\title{
Implementation of Symmetric Nonstationary Phase-Shift Wavefield Extrapolator on an Alpha Cluster
}

\author{
Yanpeng Mi and Gary F. Margrave \\ CREWES Project, Geology and Geophysics Department, The University of Calgary, \\ 2500 University Drive, Calgary, Alberta T2N 1N4, Canada \\ moceo.ucajary.ca, arrydeo.ugalaarv.ca
}

\begin{abstract}
Highly accurate seismic depth imaging (migration) is required by the oil/gas exploration industry in regions of complex geology. Fourier-domain depthimaging techniques provide the needed accuracy although the cost of computation can be large when the lateral velocity variation is rapid. Parallel computers provide an efficient solution to overcome the computational barrier of Fourier-domain imaging techniques. In this paper, a new Fourier-domain imaging technique, based on nonstationary filtering and wavefield extrapolation theories, is described. The amount of computation required for an example 2D synthetic seismic data set is analyzed. The parallel version of the algorithm is implemented using $\mathrm{C}++$ and Fortran 90 on an Alpha cluster workstation. The Message Passing Interface (MPI) library was used for data distribution and collection. The algorithm, found to be accurate but slow on serial machines, can achieve a speed acceptable for most industrial applications.
\end{abstract}

\section{Introduction}

Seismic depth imaging repositions the scattered energy from the subsurface to its correct spatial location according to a velocity model. Produced by a trueamplitude depth imaging technique, a subsurface image also carries amplitude information that is directly proportional to reflection coefficients.

Kirchhoff depth-imaging techniques are very common in the exploration industry due to robustness and high efficiency. Theoretically the method is based on Green's theorem and it is implemented by summation along iso-time surfaces computed by raytracing. Difficulties arise when it is used in regions of complex geology. Fourier-domain imaging algorithms, especially for complex media, are often slower than Kirchhoff-type techniques. However, they provide a higher accuracy solution by allowing the energy to propagate in all possible directions instead of only the Snell's law paths. Fourier-domain techniques can be made efficient by making reasonable approximations and operating in efficient data domains. 


\section{Fourier domain wavefield extrapolation}

The wavefield at depth $\mathrm{z}$ can be obtained by extrapolation the wavefield at depth 0 , given knowledge of the velocity field between 0 and $\mathrm{z}$. Consider a mono-frequency wavefield at depth $0, \varphi\left(\mathrm{k}_{\mathrm{x}}, z=0, \omega\right)$, which has been $2 \mathrm{D}$ forward Fourier transformed to frequency-wavenumber $\left(\omega, \mathrm{k}_{\mathbf{x}}\right)$ domain, the wavefield at depth $\mathrm{z}$ can be written as (Gazdag, 1978),

$$
\varphi\left(\mathrm{k}_{\mathrm{x}}, z, \omega\right)=\varphi\left(\mathrm{k}_{\mathrm{x}}, 0, \omega\right) \alpha\left(\mathrm{k}_{\mathrm{x}}, z, \omega\right)
$$

where $\alpha\left(\mathrm{k}_{\mathrm{x}}, z, \omega\right)$ is the $\left(\omega, \mathrm{k}_{\mathrm{x}}\right)$ domain wavefield extrapolator operating from 0 to $z$.

For homogeneous and isotropic media, the wavefield extrapolator is known analytically and the extrapolation step size is unlimited. For velocity $v, \alpha$ can be written as

$$
\alpha\left(\mathrm{k}_{\mathrm{x}}, z, \omega\right)=e^{ \pm i z \sqrt{\frac{\omega^{2}}{v^{2}}-k_{x}^{2}}}
$$

where the plus and minus signs denote upward and downward extrapolation when $\mathrm{z}$ axis points downward. For heterogeneous media, the extrapolation operator is known only approximately and the step size is limited. The extrapolation is done recursively with small steps, so that local wave propagation can be approximated by homogeneous and isotropic propagation theory.

There are several ways to compute an approximate wavefield extrapolator for heterogeneous media. Typical algorithms are phase-shift-plus-interpolation (PSPI) originally developed by Gazdag and Sguazzero (1984) and the split-step Fourier algorithm originally developed by Stoffa et al. (1990), which is also called the phase-screen algorithm by Wu and Huang (1992). The PSPI algorithm uses several constant velocities to produce reference extrapolated wavefields. The wavefield at any spatial location is computed by linear frequency-space $(\omega, x)$ domain interpolation between the reference wavefields according to the velocity function $v(x)$. The split-step Fourier algorithm assumes the lateral velocity variation is small so that perturbation theory applies. It first applies a homogeneous wavefield extrapolation with a constant reference velocity and then performs a $(\omega, x)$ domain slowness perturbation correction to accommodate lateral velocity variation. There are many further developments of the above algorithms in order to deal with extreme lateral velocity variation and achieve high efficiency (Jin and $\mathrm{Wu}, 1998$; Popovici, 1996; etc.) These algorithms have also been implemented on parallel computers, for example, Tanis and Stoffa (1997). 


\section{Nonstationary Fourier wavefield extrapolators}

Using a complete set of reference velocities, the PSPI algorithm becomes an integral over horizontal wave number $k_{x}$, which performs wavefield extrapolation simultaneously with an inverse Fourier transform (Margrave and Ferguson 1999a)

$$
\psi(\mathrm{x}, z, \omega)=\frac{1}{(2 \pi)^{2}} \int_{-\infty}^{+\infty} \alpha\left(\mathrm{x}_{1} \mathrm{k}_{\mathrm{x}}, z, \omega\right) \varphi\left(\mathrm{k}_{\mathrm{x}}, 0, \omega\right) \exp \left(-i \mathrm{k}_{\mathrm{x}} x\right) d \mathrm{k}_{\mathrm{x}},
$$

where $\alpha\left(\mathrm{x}, \mathrm{k}_{\mathrm{x}}, z, \omega\right)$ is the nonstationary wavefield extrapolator

$$
\alpha\left(\mathrm{x}, \mathrm{k}_{\mathrm{x}}, z, \omega\right)=e^{ \pm i z \sqrt{\frac{\omega^{2}}{v^{2}(x)} k_{x}^{2}}}
$$

$\psi(\mathrm{x}, z, \omega)$ is the $(\omega, \mathrm{x})$ domain expression of the wavefield at depth $\mathrm{z}$ and $\varphi\left(\mathrm{k}_{\mathrm{x}}, 0, \omega\right)$ is the $\left(\omega, \mathrm{k}_{\mathrm{x}}\right)$ domain expression of the wavefield at depth 0 . The meanings of plus and minus signs are defined as before. Though there is no interpolation in equation (3), the expression PSPI is used for equation (3) because it is a limiting form of Gazdag's PSPI.

Equation (3) has a complementary form, called nonstationary phase-shift (NSPS), which performs wavefield extrapolation simultaneously with a forward Fourier transform (Margrave and Ferguson, 1999a)

$$
\varphi\left(\mathrm{k}_{\mathrm{x}}, z, \omega\right)=\int_{-\infty}^{+\infty} \alpha\left(\mathrm{x}_{\mathrm{x}} \mathrm{k}_{\mathrm{x}}, z, \omega\right) \psi(\mathrm{x}, 0, \omega) \exp \left(i \mathrm{k}_{\mathrm{x}} x\right) d \mathrm{x}
$$

where $\alpha\left(\mathrm{x}, \mathrm{k}_{\mathrm{x}}, z, \omega\right)$ is expressed by equation (4).

Equation (3) can be approximately computed by matrix-vector multiplication, which can be written as

$$
\underline{\psi}_{z}=\underline{\underline{\varphi}}_{0}
$$

where $\underline{\varphi}_{0}$ and $\psi_{i}$ are column vectors representing a mono-frequency wavefield in $\left(\omega, \mathrm{k}_{\mathrm{x}}\right)$ domain at depth 0 and the extrapolated wavefield in $(\omega, \mathrm{x})$ domain at depth $\mathrm{z}$, respectively. Matrix $\boldsymbol{A}$ is the combination of the wavefield extrapolator and the simultaneous inverse Fourier-transform kernel 


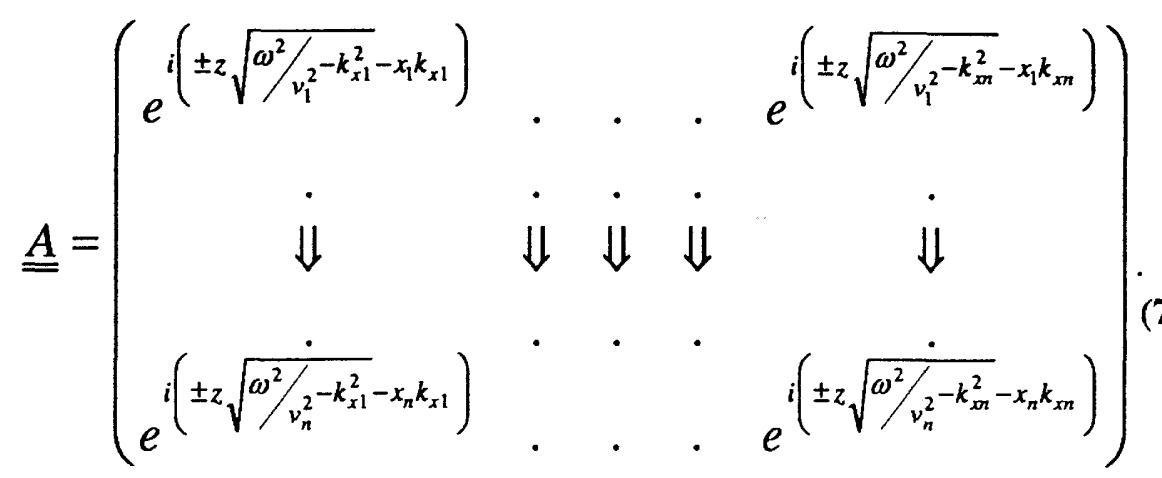

Similarly, equation (5) can be approximately written in a matrix form

$$
\underline{\varphi}_{z}=\underline{\underline{B}} \underline{\underline{w}}_{0}
$$

where $\psi_{0}$ and $\varphi_{i}$ are column vectors representing a mono-frequency wavefield in $(\omega, \mathrm{x})$ domain at depth 0 and the extrapolated wavefield in $\left(\omega, \mathrm{k}_{\mathrm{x}}\right)$ domain at depth $\mathbf{z}$, respectively. Matrix $\mathbf{B}$ is the combination of the wavefield extrapolator and the simultaneous forward Fourier-transform kernel

$$
\underline{B}=\left(\begin{array}{ccccc}
\left.e^{i\left( \pm z \sqrt{\omega^{2} / v_{1}^{2}-k_{x 1}^{2}}+x_{1} k_{x 1}\right.}\right) & & & & e^{i\left( \pm z \sqrt{\omega^{2} / v_{n}^{2-k_{x 1}^{2}}+x_{n} k_{x 1}}\right)} \\
\Downarrow & \cdot & \cdot & \cdot & \cdot \\
\Downarrow & \Downarrow & \Downarrow & \Downarrow \\
\left.e^{i\left( \pm z \sqrt{\omega^{2} / v_{1}^{2}-k_{x n}^{2}}+x_{1} k_{x n}\right.}\right) & \cdot & \cdot & \cdot & \\
& \cdot & \cdot & \cdot & \left.e^{\left( \pm z \sqrt{\omega^{2} / v_{n}^{2}-k_{x n}^{2}}+x_{n} k_{x n}\right.}\right)
\end{array}\right)
$$

Margrave and Ferguson (1999b) showed that the NSPS and PSPI can be naturally combined into a symmetric wavefield extrapolator (SNPS) by first performing NSPS for the upper half $\mathrm{z}$ and PSPI for the lower half $\mathrm{z}$ within a single step. A Taylor series derivation of PSPI and NSPS and related error analysis showed that the first-order errors of PSPI and NSPS oppose one another, so that SNPS has a smaller error and is more stable than either PSPI or NSPS alone (Margrave and Ferguson, 2000).

Prestack depth imaging of a reflector requires that the wavefield recorded on the Earth's surface be extrapolated backward and a model of the seismic source be extrapolated forward. At any particular depth, reflectivity is estimated as the ratio of the backward extrapolated recorded data (reflected wave) to the forward extrapolated source model (incident wave). To avoid a frequency dependent estimate, an average over frequency is used 


$$
\tilde{r}(\mathrm{x}, z)=\frac{1}{\left[\omega_{\max }-\omega_{\min }\right]_{\omega_{\min }}} \int_{\omega_{\mathrm{max}}}\left\{\frac{\psi^{*}{ }_{R}(\mathrm{x}, z, \omega)}{\psi^{*}(\mathrm{x}, z, \omega)}+\frac{\psi_{R}(\mathrm{x}, z, \omega)}{\psi_{S}(\mathrm{x}, z, \omega)}\right\} d \omega
$$

where $\psi_{S}$ and $\psi_{R}$ are the incident and reflected wavefields immediately above the reflector at depth $\mathrm{z}$, and $*$ denotes complex conjugation.

\section{Computational considerations for shot gather depth imaging}

Accurate depth imaging with SNPS requires that equations (3) and (5) are computed at every depth step. To study the amount of computation, a synthetic seismic data set called Marmousi (Bourgeois et al., 1991) was used. The original data set was computed by finite difference modeling. It simulates marine acquisition and has 240 shot gathers. Each shot gather has 96 traces whose offsets vary from $200 \mathrm{~m}$ to $2600 \mathrm{~m}$. Each trace has 512 samples and the sampling rate is $4 \mathrm{~ms}$. Depth imaging was done with 752 downward steps of 4 $\mathrm{m}$ each. The shot gathers were padded to 256-trace split-spread geometry to accommodate the migrated energy.

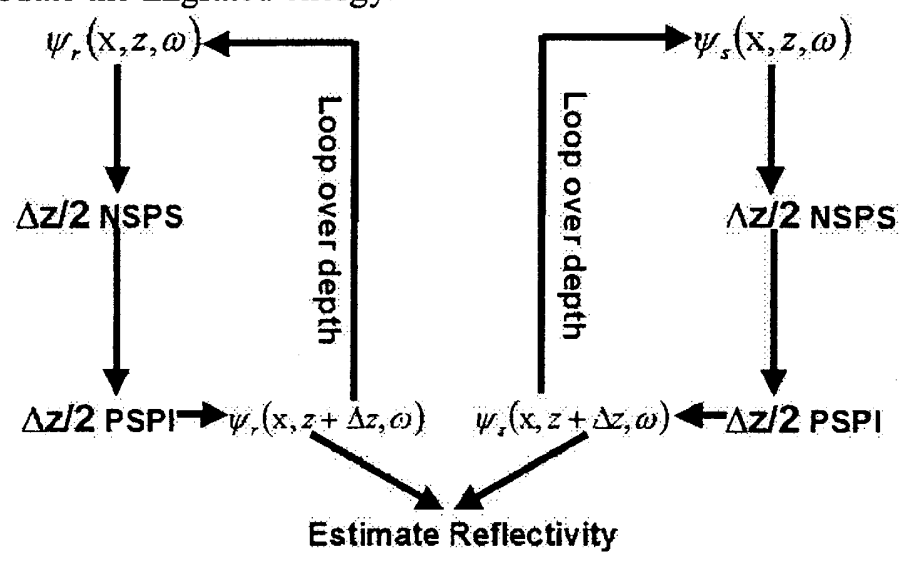

Fig. 1. Computational steps of the SNPS depth imaging algorithm.

The computing steps of SNPS are shown in Figure 1. Assume there are N traces in a shot gather and the number of frequencies needs to be migrated, $N_{\omega}$, is of the same order of $\mathrm{N}$, each step of SNPS is an $\mathrm{N}^{3}$ algorithm since each frequency needs to be computed by a matrix-vector multiplication, which is an $\mathrm{N}^{2}$ algorithm. Assume the number of depth steps is $\mathrm{N}_{\mathrm{z}}$ and the number of shots is $\mathrm{N}_{\mathrm{s}}$, the total computation required for an imaging project is of the order of $\mathrm{N}_{s} \mathrm{~N}_{\mathrm{z}} \mathrm{N}^{3}$.

It took about half an hour to migrate a single shot gather of the Marmousi data on an single-CPU, $128 \mathrm{Mb}$ memory Alpha XP 1000 workstation and the full migration of the 240 shot gathers requires 120 hours of computing time. This speed is not acceptible for industrial use though it produced a better depth 
image than commonly used algorithm in the industry. A practical approach is to compute the local approximants of the integral operators by spatial windowing. The result from each window is then collected by either a inverse spatial Fourier transform (for PSPD) or a forward spatial Fourier transform (for NSPS). Consider extrapolation from $z=0$ to depth $z$, the PSPI and NSPS integrals can be approximated by

$$
\Psi(x, z, \omega)=\sum_{j} \beta_{j}(x, \omega) \Omega_{j} \frac{1}{2 \pi} \int_{-\infty}^{\infty} \alpha_{j}\left(k_{x}, z, \omega\right) \varphi\left(k_{x}, 0, \omega\right) \exp \left(-i k_{x} x\right) d k_{x},
$$

which corresponds to a filtering-then-windowing process, and

$$
\varphi\left(k_{x}, z, \omega\right)=\sum_{j} \alpha_{j}\left(k_{x}, z, \omega\right) \int_{-\infty}^{\infty} \Psi(x, 0, \omega) \Omega_{j} \beta_{j}(x, \omega) \exp \left(i k_{x} x\right) d x
$$

which corresponds to a windowing-then-filtering process. The windowing function $\Omega_{j}$ is defined as

$$
\Omega_{j}(x)=\left\{\begin{array}{l}
1, v(x)=v_{j} \\
0, \text { otherwise }
\end{array} .\right.
$$

$\alpha_{j}\left(k_{x}, z, \omega\right)$ is defined as

$$
\alpha_{j}\left(k_{x}, z, \omega\right)=\exp \left( \pm i z \sqrt{\left(\frac{\omega}{v_{j}}\right)^{2}-k_{x}^{2}}\right)
$$

and $\beta_{f}(x, \omega)$ is $(\omega, \mathrm{x})$ domain vertical traveltime correction,

$$
\beta_{j}(\omega, x)=\exp \left( \pm i \omega z\left(v^{-1}(x)-v_{j}^{-1}\right)\right)
$$

A recursive symmetric windowed wavefield extrapolator can then be formed by combining equations (13) and (14). Computation of equation (13) is much faster than that of the SNPS integral operator since for each frequency, the $\mathrm{N}^{2}$ order matrix-vector multiplication is replaced by a Fourier transform of order $N \operatorname{logN}$. Assume the number of depth steps is $\mathrm{N}_{2}$ and the number of shots is $\mathrm{N}_{\mathrm{s}}$, and if only one reference velocity is used, the total computation required for an imaging project becomes the order of $\mathrm{N}_{\mathrm{s}} \mathrm{N}_{2} \mathrm{~N}^{2} \log \mathrm{N}$. For extremely complex media, more than one reference velocity should be used, so that the total computation becomes an algorithm of order $\mathrm{N}_{\mathrm{s}} \mathrm{N}_{\mathrm{z}} \mathrm{N}_{\mathrm{v}} \mathrm{N}^{2} \log \mathrm{N}$, where $\mathrm{N}_{\mathrm{v}}$ is the number of reference velocities.

It took about 4 minutes to migrate a shot gather on the Alpha XP 1000 workstation, on which the integral algorithm is tested. However, the image quality is slightly inferior to that produced by the SNPS integral extrapolators. 


\section{Parallel implementation on MACI Alpha Cluster}

Both the integral SNPS extrapolation algorithm and its approximations were implemented on the Multimedia Advanced Computational Infrastructure (MACI) Alpha Cluster at the University of Calgary. MACI is a collaborative project of the Universities of Alberta, Calgary, Lethbridge and Manitoba that provides high performance computing resources to Alberta researchers and educators. The MACI Alpha Cluster at the University of Calgary is part of the high-performance computing facility of the MACI project. It consists of 128 Compaq Alpha workstations and each single user can use up to 16 CPUs for a single computing task. A general network configuration of the Alpha Cluster is shown in figure 2.

XP1000s

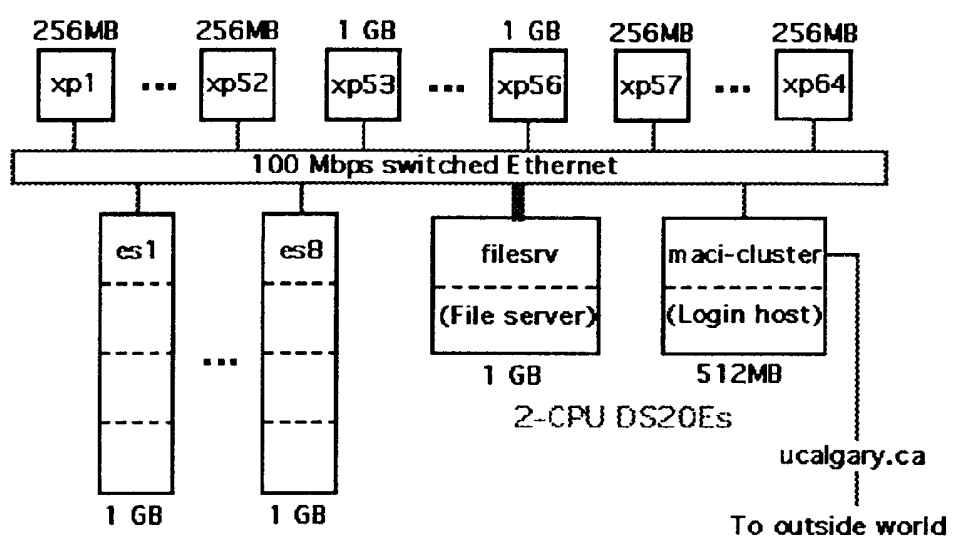

4-CPU ES4OS

Fig. 2. The general network configuration of the Alpha Cluster at the University of Calgary.

The Message Passing Interface (MPICH1.2) was used for parallel implementation. Each computing node was assigned a single shot gather migration task. The master $\mathrm{C}++$ main program performs data $\mathrm{I} / \mathrm{O}$ and parallel data distribution/collection. The shot gather imaging kernel function was written in FORTRAN 90 for high performance. It took about 1 hour to migrate the 240 shot gathers on 16 XP1000 workstations. Figure 3 shows shot gather 120 that lies in the middle of the model before and after migration. Each shot gather migration produces a local image of the subsurface and overlapping all the migrated shot gathers produces a complete subsurface image. Figure 4 shows the $0-20 \mathrm{~Hz}$ band-limited reflectivity of the Marmousi model and figure 5 shows the subsurface image generated by the integral SNPS extrapolator and that generated by its approximation. Note that the approximate algorithm produced an image only slightly inferior to that generated by the integral algorithm. The three fault planes in the middle of the model and the target flat spot at depth $2550 \mathrm{~m}$ from $\mathrm{x}=6000 \mathrm{~m}$ to $\mathrm{x}=7500 \mathrm{~m}$ are imaged well. 


\section{Conclusions and discussion}

The integral SNPS extrapolator produces a very accurate subsurface image. The approximate implementation has higher speed with very little sacrifice of image quality. The speed is directly proportional to the number of reference velocities. Direct computation of equation (3) and (5) can be made faster by utilizing the symmetric properties of the extrapolator and the forward/inverse Fourier transform kernels. Faster while less accurate numerical algorithms to compute the square root and exponential function can also be used to improve the computing performance.

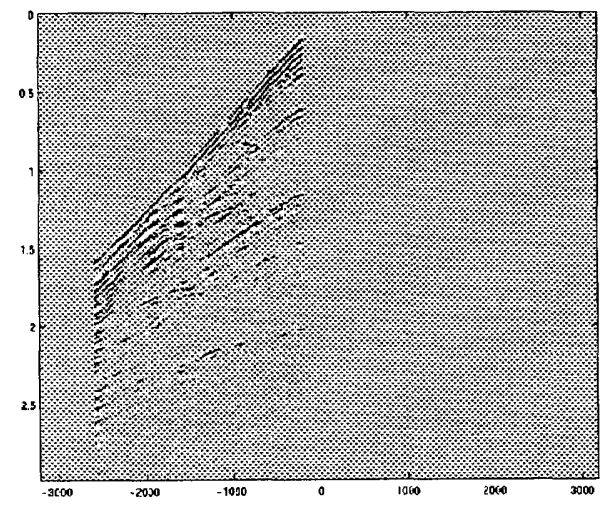

(a)

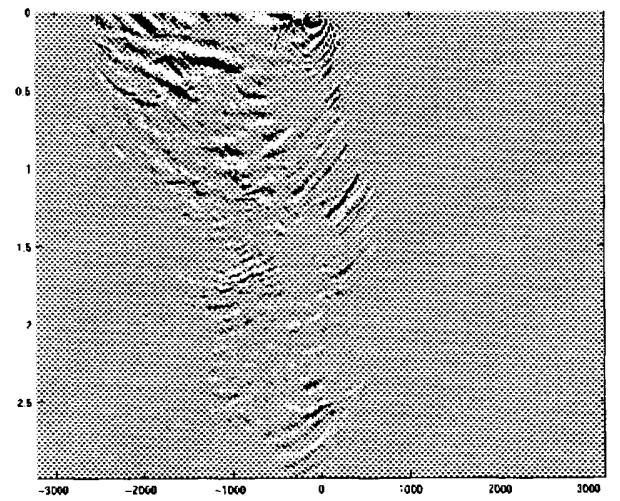

(b)

Fig. 3. Shot gather 120 before (a) and after migration (b).

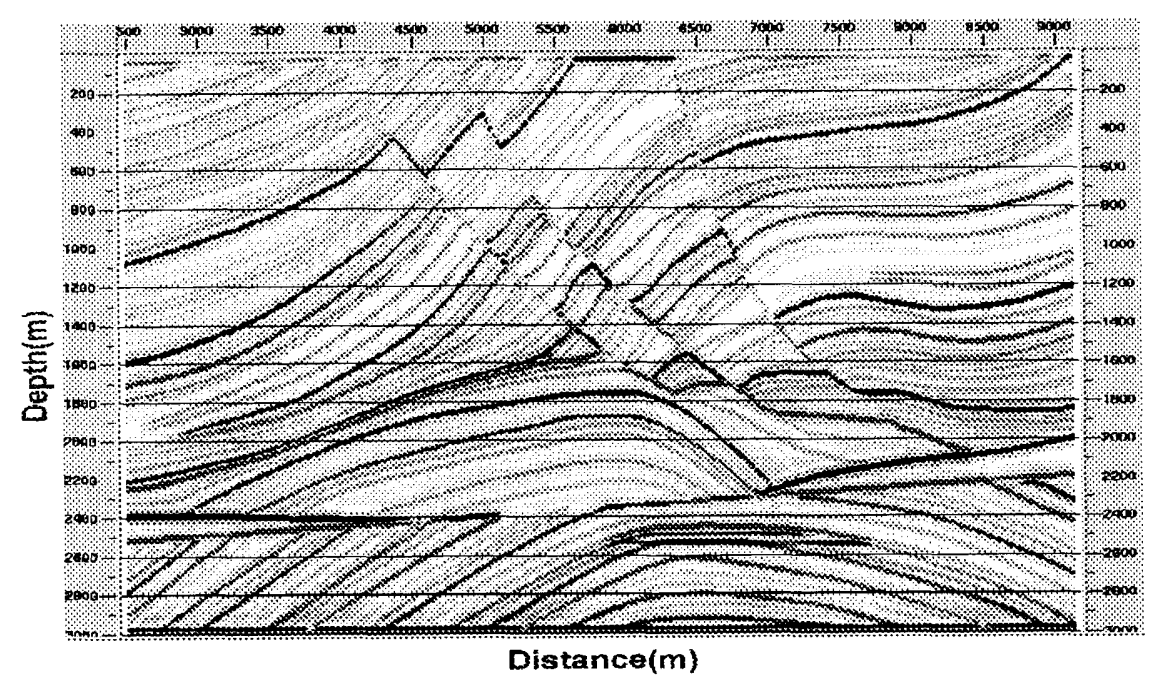

Fig. 4. 0-20 Hz band-limited reflectivity of the Marmousi model. 


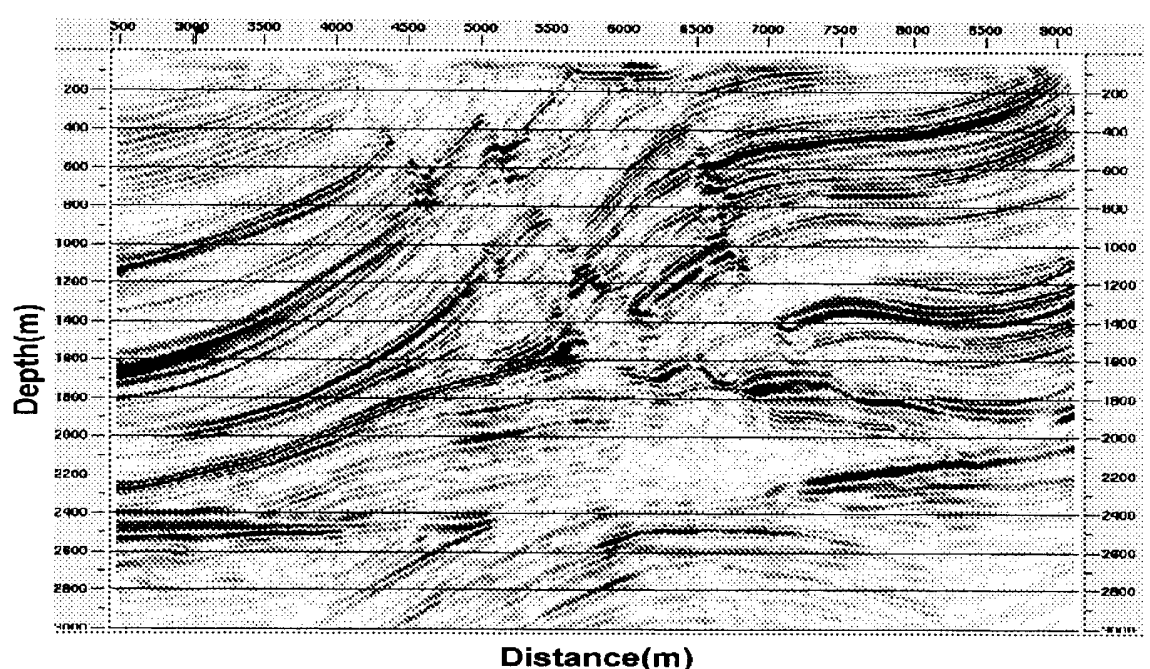

(a)

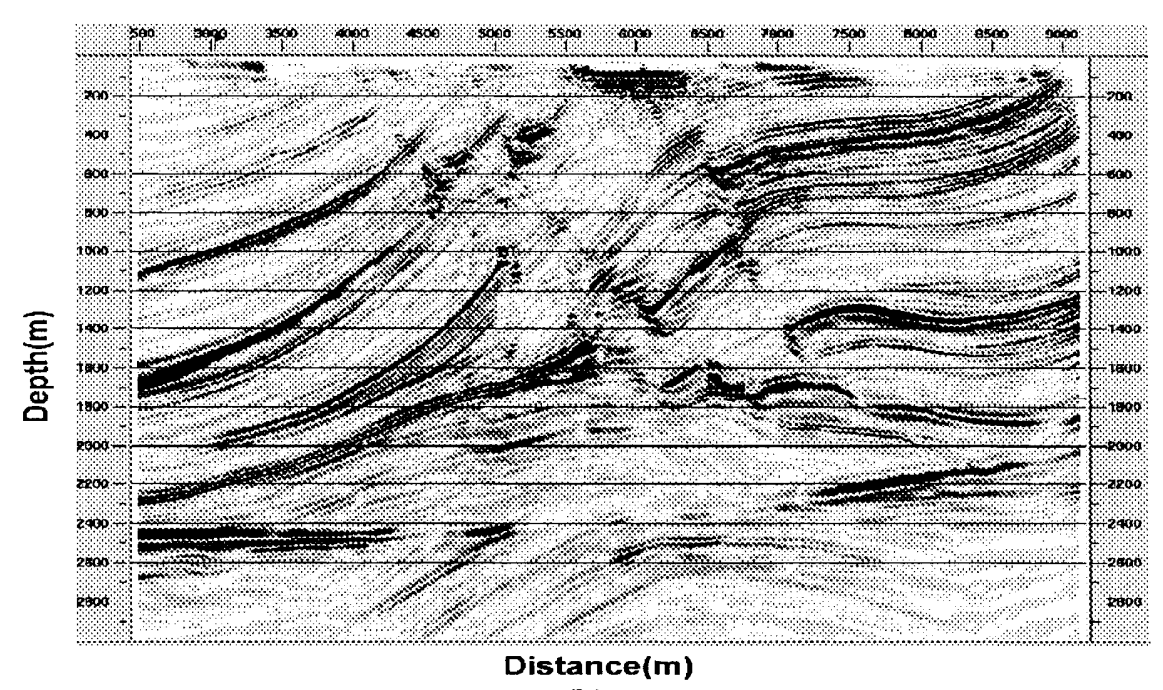

(b)

Fig. 5. (a) subsurface image produced by integral SNPS extrapolator; (b) subsurface image produced by the approximate algorithm. Only frequency content of $5-40 \mathrm{~Hz}$ was migrated. 


\section{References}

Bourgeois, A., Bourget, M., Lailly, P., Poulet, M., Ricarte, P., and Versteeg, R., 1991, Marmousi, model and data: in The Marmousi experience, EAGE, 5 - 9.

Ferguson, R. and Margrave, G., 1999, A practical implementation of depth migration by nonstationary phase shift: Annual Meeting Abstracts, Society Of Exploration Geophysicists, 1370-1373.

Gazdag, J., 1978, Wave equation migration with the phase-shift method, Geophysics, $43,1342-1351$.

Gazdag, J. and Sguazzero, P., 1984, Migration of seismic data by phase shift plus interpolation, Geophysics, 49, 124-131.

Jin, S. and Wu, R., 1998, Depth migration using the windowed generalized screen propagators: Annual Meeting Abstracts, Society Of Exploration Geophysicists, 1843-1846.

Margrave, G. F. and Ferguson, R. J., 1999a, Wavefield extrapolation by nonstationary phase shift: Geophysics, 64, no. 04, 1067-1078.

Margrave, G. and Ferguson, R., 1999b, An explicit, symmetric wavefield extrapolator for depth migration: Annual Meeting Abstracts, Society Of Exploration Geophysicists, 1461-1464.

Margrave, G. and Ferguson, R., 2000, Taylor series derivation of nonstationary wavefield extrapolators: Annual Meeting Abstracts, Society of Exploration Geophysicists, 834-837

Popovici, A. M., 1996, Prestack migration by split-step DSR: Geophysics, 61, no. 05, 1412-1416.

Stoffa, P. L., Fokkema, J. T., de Luna Freire, R. M. and Kessinger, W. P., 1990, Splitstep Fourier migration: Geophysics, 55, 410-421.

Tanis, M. C. and Stoffa, P. L., 1997, Parallel implementation of 3-D split-step Fourier depth migration algorithm on T3E: Annual Meeting Abstracts, Society Of Exploration Geophysicists, 1433-1436.

Wu, R. and Huang, L., 1992, Scattered field calculation in heterogeneous media using phase-screen propagation: Annual Meeting Abstracts, Society Of Exploration Geophysicists, 1289-1292. 